\title{
Persistent SAR Change Detection with Posterior Models
}

\author{
Gregory E. Newstadt ${ }^{a}$, Edmund G. Zelnio ${ }^{b}$, and Alfred O. Hero III ${ }^{a}$ \\ ${ }^{a}$ Electrical Engineering and Computer Science, University of Michigan, Ann Arbor, MI 48109, \\ USA; \\ ${ }^{b}$ Air Force Research Laboratory, Wright Patterson Air Force Base, OH, 45433, USA
}

\begin{abstract}
This paper develops a hierarchical Bayes model for multiple-pass, multiple antenna synthetic aperture radar (SAR) systems with the goal of adaptive change detection. The model is based on decomposing the observed data into a low-rank component and a sparse component, similar to Robust Principal Component Analysis, previously developed by Ding, He, and Carin ${ }^{1}$ for E/O systems. The developed model also accounts for SAR phenomenology, including antenna and spatial dependencies, speckle and specular noise, and stationary clutter. Monte Carlo methods are used to estimate the posterior distribution of the variables in the model. The performance of the proposed method is analyzed using synthetic images, and it is shown that the performance is robust to a large space of operating characteristics without extensive tuning of hyperparameters. Finally, the method is applied to measured SAR data, providing competitive results compared to standard methods with the additional benefits of uncertainty characterization through a posterior distribution, explicit estimates of both foreground and background components, and flexibility in including other sources of information.
\end{abstract}

Keywords: synthetic aperture radar, change detection, hierarchical Bayes model.

\section{INTRODUCTION}

Synthetic aperture radars (SAR) have traditionally been used to image stationary or slow-moving targets in a region of interest. By integrating radar pulses from spatially diverse points, SAR images lead to 2- or 3dimensional images with much finer resolutions than other airborne radar operating modes (in particular, MTI) due to the ability to use long integration times. However, the situation becomes complex when considering moving targets, which can cause phase errors in the reconstruction of SAR images. This leads to well-known defocusing and displacement of the targets' energy. ${ }^{2-4}$ Moreover, stationary clutter can often mask the target energy, further reducing the detection probability and estimation accuracy of moving targets. ${ }^{5}$

There has been significant research into exploiting multiple channels in order to extract the moving target from the background image. Soumekh ${ }^{6}$ shows that with a monopulse radar that combines a monostatic and bistatic SAR signal, all stationary targets can be removed from this signal with a simple difference image, leaving just the moving targets (albeit still defocused and displaced). In practice, the multiple antenna platforms are not perfectly calibrated, which can significantly reduce the performance. Signal subspace processing ${ }^{7}$ (SSP) addresses this problem by proposing an adaptive blind calibration technique of the two channels that assumes that the signals are related through a locally spatially invariant filter. Ender also considers using multiple channels for SAR detection of moving targets through space-time adaptive processing ${ }^{5}$ (STAP), which adaptively estimates the clutter covariance matrix in order to separate moving targets from the background. Both SSP and STAP share the properties of being adaptive to the observed signals, as well as exploiting the fact that clutter energy is embedded in a low dimensional subspace. This is a principal fact that is further exploited by the models developed in this paper.

Persistent monitoring of an area provides additional dependencies between observed signals. Non-coherent change detection algorithms are commonly used to compare magnitudes between reference and test images. In many cases, the reference image may be the simple average of previous passes that have been rotated and aligned. For this work, it is assumed that all of the signals have been registered to each other, though this

Further author information: (Send correspondence to Gregory E. Newstadt at newstage@umich.edu) This document has been cleared for public release, document number 88 ABW-11-2836.

Algorithms for Synthetic Aperture Radar Imagery XVIII, edited by Edmund G. Zelnio, Frederick D. Garber, Proc. of SPIE Vol. 8051, 80510R · C 2011 SPIE · CCC code: 0277-786X/11/\$18 · doi: 10.1117/12.888956 
task is not trivial. However, by introducing temporal dependencies, one has to be careful to account for other significant error sources, including the existence of targets in previous passes and angular scintillation (glint). In particular, glint can considerably degrade the signal quality, though it is well known that glint has a large angular dependence. ${ }^{8}$

Erten et. al. provides a statistical analysis for SAR change detection with multiple channels. ${ }^{9}$ They proposed a change detection algorithm based on mutual information (a special case of KL-divergence) that outperforms classical correlation-based change detection. In our work, we propose a hierarchical Bayes model that exploits the dependencies among multiple antennas and multiple passes, as well as inherent spatial dependencies in both stationary and moving targets. Our approach is closely related to Bayesian Robust Principal Component Analysis, ${ }^{1}$ which separates the observed signal matrix as

$$
Y=L+S+E
$$

for low-rank matrix, $L$, sparse matrix, $S$, and error matrix, $E$. This paper shows that SAR signals can be decomposed similarly by replacing the low-rank matrix, $L$, with a low-rank subspace. Sparseness is promoted through priors on the parameters of interest, in a manner similar to relevance vector machines and Bayesian approaches to compressive sensing. ${ }^{10-12}$

The first contribution of this paper is a SAR image model for persistent SAR imagery, where persistence imaging is accomplished by multiple passes of the radar platform. The second contribution of this paper is a Gibbs sampling scheme for efficient estimation of the posterior distribution. The rest of the paper is organized as follows. The description of the model assumptions is given in Section 2. Section 3 outlines notation that is commonly used throughout the paper. The hierarchical Bayes model for SAR imagery is given in Section 4. Section 5 discusses the Monte Carlo implementation for posterior inference on the model. Performance of the model and inference is analyzed with both synthetic and measured SAR imagery in Section 6. Finally, conclusions and future work are provided in Section 7.

\section{MODEL ASSUMPTIONS}

The following is a list of assumptions that we make for the proposed SAR image model:

- Phase histories are collected from a monopulse radar system with 1 transmit antenna and $K-1$ receive antennas.

- The phase histories have been aligned so that the $j$-th pulse of each phase history corresponds to the same phase center.

- Images are formed from phase histories over a discrete set of pixels, indexed by $(p)$ for the $p$-th pixel.

- $P$ passes of the radar platform are available, with perfectly aligned k-spaces to allow for independent identically distributed observations from pass to pass (up to a calibration constant.)

- Images are formed with overlapping azimuth ranges. For example, image 1 may contain azimuth angles 0 to 6 degrees, while image 2 contains azimuth angles 1 to 7 degrees.

- All images have the same azimuth extent (i.e., integration length), and can be indexed by their center angle. This center angle is isomorphic to a discrete set, $\{1,2, \ldots, M\}$, and is equivalent for all $P$ passes.

\section{NOTATION}

The following is a list of notation used throughout the paper:

- Count variables

- $P$ : Number of passes of the radar platform. 
- F: Number of sequential frames per pass (possibly overlapping and isomorphic to center azimuth angle.)

- $K$ : Number of phase centers (i.e., number of antennas).

- Pixel variables, $I_{k, f, i}(p)$ - Complex value for pixel $p$, frame $f$, pass $i$, and antenna $k$.

$-I_{k, f, i}(p)=H_{k, f, i} \otimes B_{f, i}(p)+T_{k, f, i}(p)+V_{k, f, i}(p)$, where

$* H_{k, f, i}(p)$ : Antenna gain and registration filter.

$* B_{f, i}(p)$ : Background component.

$* T_{k, f, i}(p)$ : Foreground (target) component.

* $V_{k, f, i}(p)$ : Noise component.

- : Background component, $B_{f, i}(p)=S_{f}(p)+X_{f, i}(p)+\delta_{f}(p) Y_{f, i}(p)$

* $S_{f}(p)$ : Stationary (low-rank) component.

$* X_{f, i}(p)$ : Speckle noise component (low-amplitude).

* $Y_{f, i}(p)$ : Specular noise component (high-amplitude).

$* \delta_{f}(p)$ : Specular noise indicator (sparse).

- Foreground component, $T_{k, f, i}(p)=D_{f, i}(p) M_{k, f, i}(p)$

* $M_{k, f, i}(p)$ : Target component (low-amplitude).

* $D_{f, i}(p)$ : Target indicator (sparse).

Other distributional parameters will be defined in the body of the model description below.

\section{SAR IMAGE MODEL}

We proposes a decomposition of SAR images into a background component and a foreground component, along with zero-mean additive noise. The decomposition is similar to robust Bayesian principal component analysis. ${ }^{1}$ We lump the errors related to spatial/spectral registration, calibration of antenna gains, and image reconstruction into an unknown filter function $H_{k, f, i}$. This is similar to the model used in signal subspace projection. ${ }^{7}$ This leads to the basic image model:

$$
I_{k, f, i}(p)=H_{k, f, i} \otimes B_{f, i}(p)+T_{k, f, i}(p)+V_{k, f, i}(p)
$$

where $H_{k, f, i}$ is a spatially varying filter, $B_{f, i}$ is the background component, $T_{k, f, i}$ is the foreground component, $V_{k, f, i}$ is zero-mean additive noise, and $(p, k, f, i)$ index the pixel, antenna, frame, and pass, respectively. The remainder of this section will focus on each component separately.

\subsection{Image reconstruction filter, $H_{k, f, i}$}

Ranney and Soumekh ${ }^{7}$ propose that reference and test SAR imagery can be related by a spatially varying filter. For 2-dimensional images, this is equivalent to:

$$
I_{k, f, i}(x, y)=\sum_{m=-n_{x}}^{n_{x}} \sum_{n=-n_{y}}^{n_{y}} H_{k, f, i}^{g}(m, n) B_{k_{0}, f_{0}, i_{0}}\left(x-m \Delta_{x}, y-n \Delta_{y}\right)+T_{k, f, i}(x, y)+V_{k, f, i}(x, y)
$$

for all pixels $p=(x, y) \in Z_{g}$, where $Z_{g}$ is a subset of pixel locations. We will assume that images are registered well to each other, so that $n_{x}=n_{y}=0$ and

$$
I_{k, f, i}(x, y)=H_{k, f, i}^{g} B_{k_{0}, f_{0}, i_{0}}(x, y)+T_{k, f, i}(x, y)+V_{k, f, i}(x, y)
$$

for all pixels $p=(x, y) \in Z_{g}$. Equivalently,

$$
\begin{aligned}
H_{k, f, i}(p) & =H_{k, f, i}^{g}, \forall p \in Z_{g} \\
I_{k, f, i}(p) & =H_{k, f, i}(p) B_{f_{0}, i_{0}}(p)+T_{k, f, i}(p)+V_{k, f, i}(p)
\end{aligned}
$$


Finally, it is assumed that

$$
\begin{aligned}
H_{k, f, i}^{g} & \sim \mathcal{C N}\left(1, \sigma_{h}^{2}\right), \forall g \\
\sigma_{h}^{-2} & \sim \operatorname{Gamma}\left(c_{h}, d_{h}\right)
\end{aligned}
$$

where $\mathcal{C N}\left(\mu, \sigma^{2}\right)$ is the complex normal distribution with mean $\mu$ and covariance $I \sigma^{2}$. As in Tipping, ${ }^{10}$ we fix the hyperparameters $c_{h}=d_{h}=10^{-6}$ to promote non-informative priors.

4.2 Background component, $B_{f, i}(p)$

The background component contains the low-rank features of the image as well as the perturbations caused by speckle and specular noise. Here we propose

$$
B_{f, i}(p)=S_{f}(p)+X_{f, i}(p)+\delta_{f}(p) Y_{f, i}(p)
$$

where $S_{f}(p)$ is low-rank, $X_{f, i}(p)$ is speckle noise, $Y_{f, i}(p)$ is specular noise, and $\delta_{f}(p)$ is an indicator of specular noise. Note that the background component has no dependence on antenna.

\subsubsection{Low-rank component, $S_{f}(p)$}

Pixels in a SAR image tend to have strong spatial dependence, depending on the type of object present in an area. For example, roads tend to have both low amplitudes and low variances, vegetation tend to have low amplitudes and high variance, and buildings tend to have high amplitudes. To account for this spatial dependence, we propose the following statistical model

$$
\begin{aligned}
S_{f}(p) & \sim \mathcal{C N}\left(0, \sigma_{s}^{2}(j)\right), \forall p \in Q_{j} \\
\sigma_{s}^{-2}(j) & \sim \operatorname{Gamma}\left(c_{s}, d_{s}\right)
\end{aligned}
$$

where $Q_{j}$ is a subset of pixel locations. As before, $c_{s}=d_{s}=10^{-6}$. To further reduce the rank of the stationary component, one can suppose that each subregion of pixels, $Q_{j}$, can be identified by one of $E$ distributions (for example, roads/trees/buildings). Under this assumption, define

$$
\begin{aligned}
\vec{d}(j)=\left\{d_{n}(j)\right\}_{n=1}^{E} & \sim \operatorname{Multinomial}\left(1 ; q_{1}, q_{2}, \ldots, q_{E}\right) \\
\left\{q_{n}\right\}_{n=1}^{D} & \sim \operatorname{Dirichlet}\left(\alpha_{d}(1), \alpha_{d}(2), \ldots, \alpha_{d}(E)\right)
\end{aligned}
$$

where $q_{n}$ is the prior probability of the $n$-th region type. Note that $\vec{d}(j)$ contains exactly one entry with value equal to unity. Then, the rank-restricted model is given by

$$
\begin{aligned}
\sigma_{s}^{2}(j) & =\sum_{n=1}^{D} d_{n}(j) Z_{n} \\
Z_{n}^{-1} & \sim \operatorname{Gamma}\left(c_{s}, d_{s}\right)
\end{aligned}
$$

In the prior-knowledge free case, the authors propose that $\alpha_{d}(l)=\alpha_{d} \ll 1, l=1,2, \ldots, E$, and $E$ is large enough to contain all possible region types. For the measured SAR data in this work, the parameters were chosen as $E=10$ and $\alpha_{d}=1 /\left(N_{x} N_{y}\right)$, where $N_{x}, N_{y}$ are the number of pixels in the reconstructed image in the $x$ - and $y$ directions.

\subsubsection{Speckle noise, $X_{f, i}(p)$}

Speckle noise is caused by random fluctuations in the complex phase history of stationary objects. For example, slowly moving blades of grass will cause slightly different returns when viewed at different times. These variations are assumed to be low in amplitude, and are modeled as

$$
\begin{aligned}
X_{f, i}(p) & \sim \mathcal{C N}\left(0, \sigma_{\text {speckle }}^{2}\right) \\
\sigma_{\text {speckle }}^{-2} & \sim \operatorname{Gamma}\left(c_{\text {speckle }}, d_{\text {speckle }}\right)
\end{aligned}
$$

If desired, one can impose a spatial dependence on speckle noise similar to $\sigma_{s}^{2}(j)$. Once again, as suggested by Tipping, ${ }^{10}$ we fix $c_{\text {speckle }}=d_{\text {speckle }}=10^{-6}$. 


\subsubsection{Specular noise, $\delta_{f}(p) Y_{f, i}(p)$}

Specular noise can cause large-amplitude variations in the image. However, specular noise is highly dependent on the azimuth angle and will be sparse for small to moderate integration lengths. Thus, specular noise is modeled as

$$
\begin{aligned}
Y_{f, i}(p) & \sim \mathcal{C N}\left(0, \sigma_{\text {specular }}^{2}\right) \\
\delta_{f}(p) & \sim \operatorname{Bernoulli}\left(\pi_{\text {specular }}\right) \\
\sigma_{\text {specular }}^{-2} & \sim \operatorname{Gamma}\left(c_{\text {specular }}, d_{\text {specular }}\right) \\
\pi_{\text {specular }} & \sim \operatorname{Beta}\left(\alpha_{\text {specular }}, \beta_{\text {specular }}\right)
\end{aligned}
$$

The authors suggest that $c_{\text {specular }}=d_{\text {specular }}=10^{-6}$ and $\alpha_{\text {specular }} /\left(\alpha_{\text {specular }}+\beta_{\text {specular }}\right) \ll 1$. In this paper, we set $\alpha_{\text {specular }}=1 / F$ and $\beta_{\text {specular }}=1-\alpha_{\text {specular }}$. It is also possible to impose a spatial dependence on $\delta_{f}(p)$, since specular noise often affects multiple pixels simultaneously (for example, glints on the sides of buildings, which tend to face the same direction).

\subsection{Foreground component, $T_{k, f, i}$}

The foreground component of the SAR image contains the targets (possibly stationary) that are of primary interest to the change detection algorithm. For sufficiently high PRF, target signatures will vary smoothly over sequential frames. Thus, it is logical to impose a Markov dependency on the pixels from sequential frames. This work adopts the sparse pixel model from Bayesian Robust PCA. ${ }^{1}$ In particular, define the following:

$$
T_{k, f, i}(p)=D_{f, i}(p) M_{k, f, i}(p)
$$

where

$$
\begin{aligned}
M_{k, f, i}(p) & \sim \mathcal{C N}\left(0, \sigma_{\text {target }}^{2}\right) \\
D_{f, i}(p) & \sim \operatorname{Bernoulli(}\left(\pi_{\text {target }}(p ; f, i)\right) \\
\sigma_{\text {target }}^{-2} & \sim \operatorname{Gamma}\left(c_{\text {target }}, \beta_{\text {target }}\right) \\
\pi_{\text {target }}(p ; f, i) & \sim\left\{\begin{array}{cc}
\operatorname{Beta}\left(\alpha_{\text {target }}, \beta_{\text {target }}\right) & f=0 \\
\operatorname{Beta}\left(\alpha_{\text {targh }}^{\text {high }}, \beta_{\text {targhet }}^{\text {high }}\right) & f>0, N_{f-1, i}(p)>\varepsilon \\
\operatorname{Beta}\left(\alpha_{\text {target }}^{\text {low }}, \beta_{\text {target }}^{\text {low }}\right) & f>0, N_{f-1, i}(p) \leqslant \varepsilon
\end{array}\right.
\end{aligned}
$$

and $N_{f, i}\left(p_{0}\right)$ is the number of neighbors $p$ of pixel $p_{0}$ with $D_{f, i}(p)=1$. Further dependencies can be imposed on $\pi_{\text {target }}(p ; f, i)$ to include SAR-specific phenomenology. For example, target signatures tend to be blurred perpendicular to the platform trajectory. ${ }^{3}$

\subsection{Noise component}

The noise component is assumed to be complex normally distributed:

$$
\begin{aligned}
V_{k, f, i}(p) & \sim \mathcal{C N}\left(0, \sigma_{v}^{2}\right) \\
\sigma_{v}^{-2} & \sim \operatorname{Gamma}\left(c_{v}, d_{v}\right)
\end{aligned}
$$

\section{POSTERIOR INFERENCE}

In this hierarchical Bayesian model, the hyper-parameters are conjugate to the prior distributions of the variables of interest. Thus, the computation of the posterior distribution is greatly simplified. This work provides a Gibbs sampling scheme for estimating the posterior distribution given the images. The Gibbs sampler requires an initial estimate of the variables and a large number of $N_{\text {burn-in }}$ samples for the Markov chain to become stable. Then $N_{\text {samples }}$ are collected, presumably from the posterior distribution. For images $I$ and initial estimate $\Theta_{0}$, the Gibbs sampler is summarized in Algorithm 1. The algorithm is given for the situation where $\sigma_{s}^{2}(j)$ can very over all subregions, $Q_{j}$, though the alteration to enforce low-rank requires only a simple change to the algorithm. 


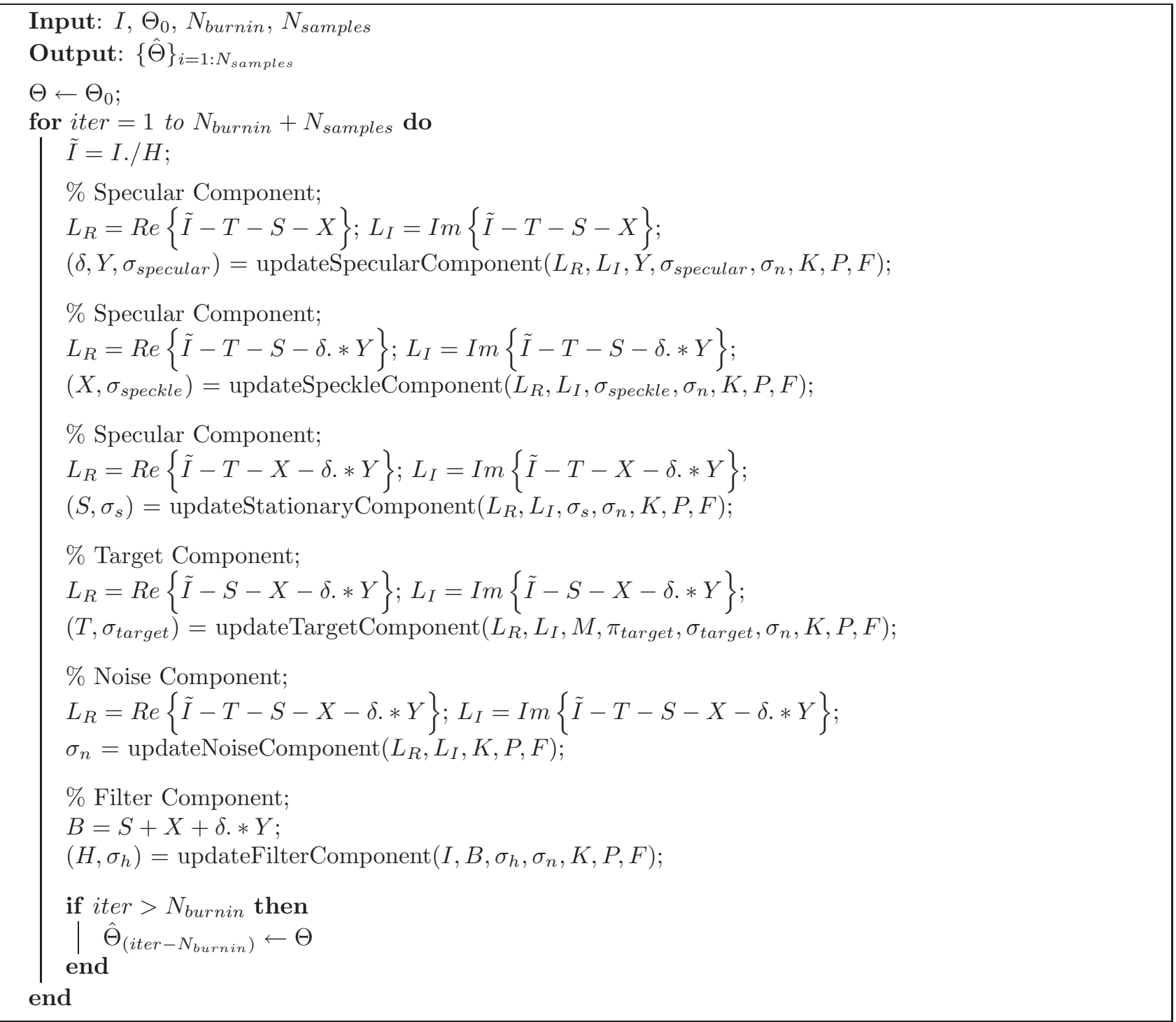

Algorithm 1: Pseudocode for Gibbs Sampling for Posterior Inference 
Input: $L_{R}, L_{I}, Y, \sigma_{\text {specular }}, \sigma_{n}, K, P, F$

Output: $\delta, Y, \sigma_{\text {specular }}$

$\%$ Indicator variables;

$\sigma_{0}^{2}=\sigma_{n}^{2}$

$\sigma_{1}^{2}=\sigma_{\text {specular }}^{2}+\sigma_{n}^{2}$

foreach $f, p$ do

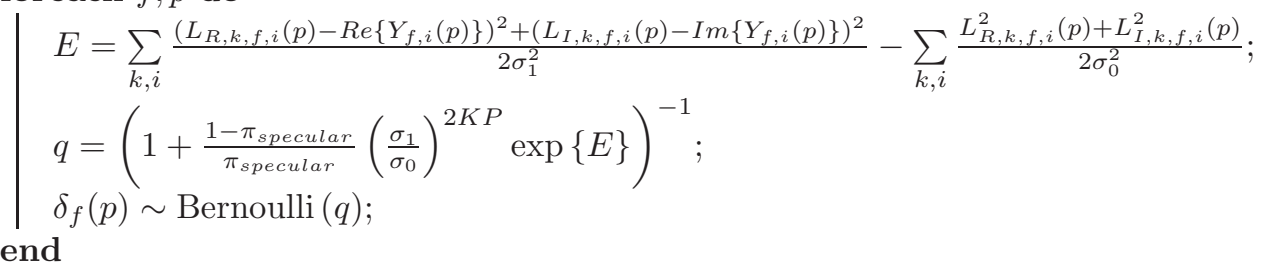

$\%$ Specular noise values;

foreach $p, f, i$ do

$\mid \begin{aligned} & \alpha=\frac{\sigma_{\text {specular }}^{2}}{K \sigma_{\text {specular }}+\sigma_{n}^{2}} ; \mu=\alpha \sum_{k}\left(L_{R, k, f, i}(p)+j L_{I, k, f, i}(p)\right) ; \sigma^{2}=\alpha \sigma_{n}^{2} \\ & Y_{f, i}(p) \sim \mathcal{C N}\left(\mu, \sigma^{2}\right)\end{aligned}$

$\%$ Hyperparameters;

$c_{1}=c_{\text {specular }}+P\left(\sum_{p, f} \delta_{f}(p)\right) ; d_{1}=d_{\text {specular }}+\sum_{p, f, i}\left(\operatorname{Re}\left\{\delta_{f}(p) Y_{f, i}(p)\right\}^{2}+\operatorname{Im}\left\{\delta_{f}(p) Y_{f, i}(p)\right\}^{2}\right) ;$

$\sigma_{\text {specular }}^{-2} \sim \operatorname{Gamma}\left(c_{1}, d_{1}\right)$;

Function updateSpecularComponent $\left(L_{R}, L_{I}, Y, \sigma_{\text {specular }}, \sigma_{n}, K, P, F\right)$

Input: $L_{R}, L_{I}, \sigma_{\text {speckle }}, \sigma_{n}, K, P, F$

Output: $X, \sigma_{\text {speckle }}$

$\%$ Speckle noise values;

foreach $p, f, i$ do

$\mid \begin{aligned} & \alpha=\frac{\sigma_{\text {speckle }}^{2}}{K \sigma_{\text {speckle }}^{+2} \sigma_{n}^{2}} ; \mu=\alpha \sum_{k}\left(L_{R, k, f, i}(p)+j L_{I, k, f, i}(p)\right) ; \sigma^{2}=\alpha \sigma_{n}^{2} \\ & \quad X_{f, i}(p) \sim \mathcal{C N}\left(\mu, \sigma^{2}\right) ;\end{aligned}$

$\%$ Hyperparameters;

$c_{1}=c_{\text {speckle }}+P F N_{x} N_{y} ; d_{1}=d_{\text {speckle }}+\sum_{p, f, i}\left(\operatorname{Re}\left\{X_{f, i}(p)\right\}^{2}+\operatorname{Im}\left\{X_{f, i}(p)\right\}^{2}\right) ;$

$\sigma_{\text {speckle }}^{-2} \sim \operatorname{Gamma}\left(c_{1}, d_{1}\right)$

Function updateSpeckleComponent $\left(L_{R}, L_{I}, \sigma_{\text {speckle }}, \sigma_{n}, K, P, F\right)$ 
Input: $L_{R}, L_{I}, \sigma_{s}, \sigma_{n}, K, P, F$

Output: $S, \sigma_{s}$

$\%$ Stationary component values;

foreach $Q_{j}$ do

foreach $p, f$ do

$\alpha=\frac{\sigma_{s}^{2}(j)}{K P \sigma_{s}^{2}(j)+\sigma_{n}^{2}} ; \mu=\alpha \sum_{k, i}\left(L_{R, k, f, i}(p)+j L_{I, k, f, i}(p)\right) ; \sigma^{2}=\alpha \sigma_{n}^{2} ;$

$S_{f}(p) \sim \mathcal{C N}\left(\mu, \sigma^{2}\right)$

end

$\%$ Hyperparameters;

$c_{1}=c_{s}+F\left|Q_{j}\right| ; d_{1}=d_{s}+\sum_{f, p \in Q_{j}}\left(\operatorname{Re}\left\{S_{f}(p)\right\}^{2}+\operatorname{Im}\left\{S_{f}(p)\right\}^{2}\right) ;$

$\sigma_{s}^{-2}(j) \sim \operatorname{Gamma}\left(c_{1}, d_{1}\right)$

end

Function updateStationaryComponent $\left(L_{R}, L_{I}, \sigma_{s}, \sigma_{n}, K, P, F\right)$

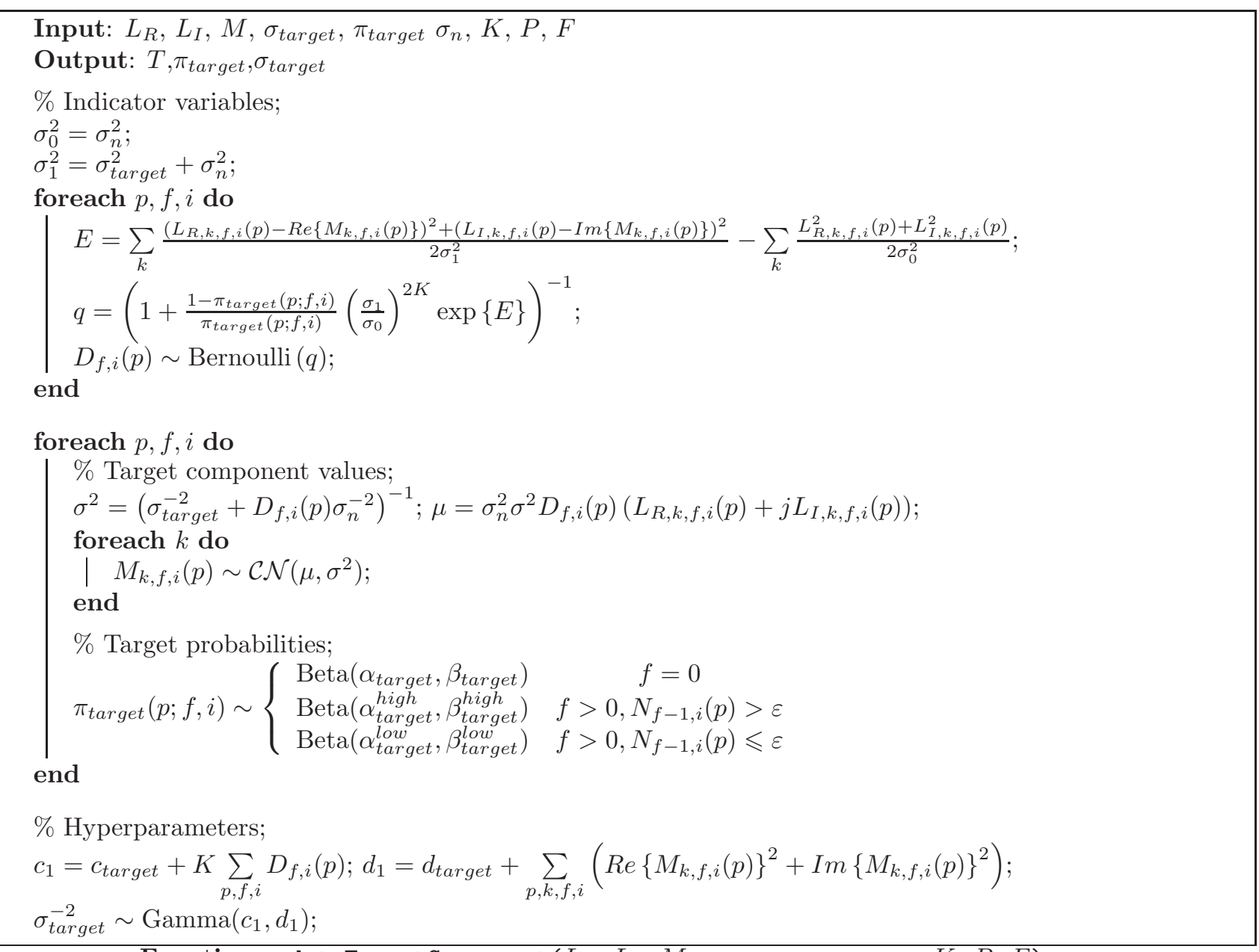

Function updateTargetComponent $\left(L_{R}, L_{I}, M, \pi_{\text {target }}, \sigma_{\text {target }}, \sigma_{n}, K, P, F\right)$ 


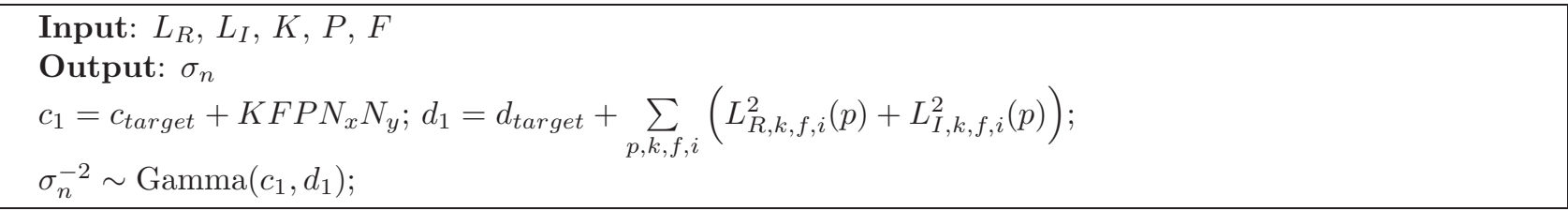

Function updateNoiseComponent $\left(L_{R}, L_{I}, K, P, F\right)$

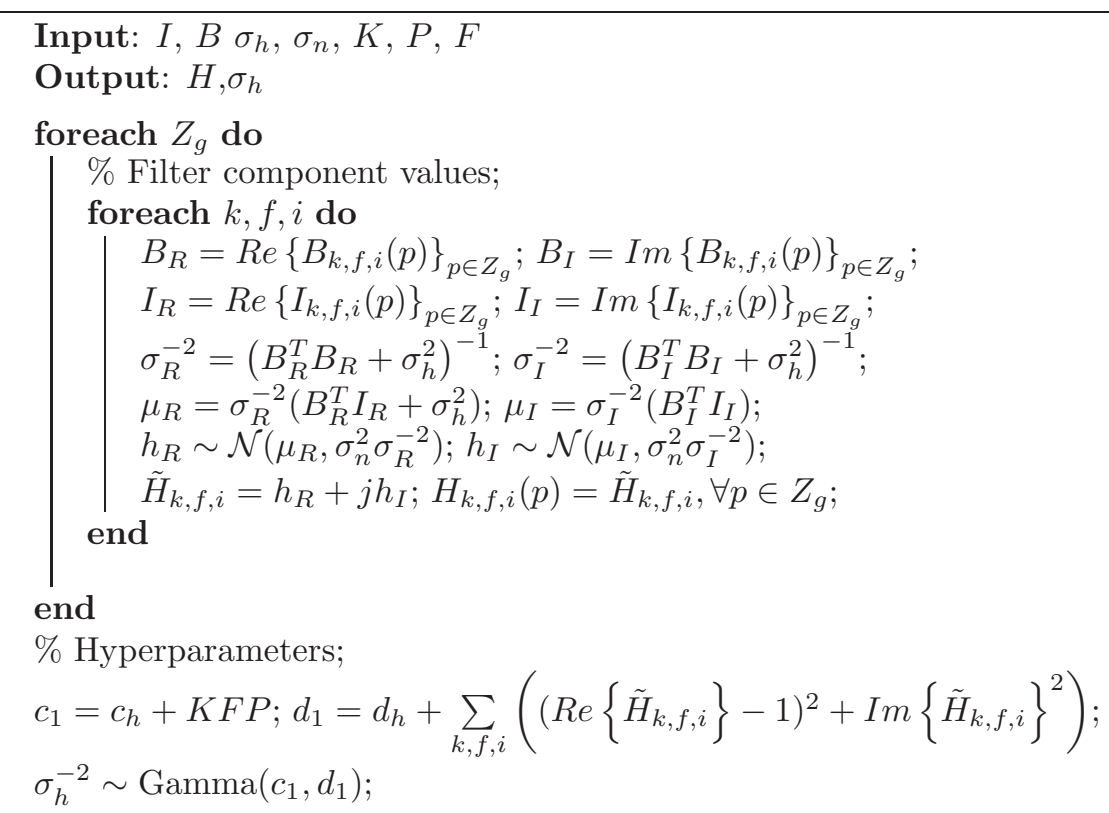

Function updateFilterComponent $\left(I, B, \sigma_{h}, \sigma_{n}, K, P, F\right)$

\section{PERFORMANCE ANALYSIS}

\subsection{Simulated SAR imagery}

To evaluate the performance of our proposed hierarchical Bayesian model and the associated Gibbs sampler, simulated imagery was created to simulate different SAR operating conditions. This included variations in the underlying model, such as specular noise variance, $\sigma_{\text {specular }}^{2}$, and target variance, $\sigma_{\text {target }}^{2}$, as well as variations in the available data, such as number of passes, $P$, and number of phase centers, $K$. Each image contained $100 \mathrm{x}$ 100 pixels, and was separated into three region types of different variances (i.e., $E=3$ ). Images were corrupted by additive noise $\left(\sigma_{n}^{2}=0.1\right)$ and complex reconstruction filter, $H\left(\sigma_{h}^{2}=0.1\right)$. A single moving target signature is present in pass with random initial starting location. Furthermore, the target signature was varied smoothly over sequential frames. Specular noise was added to a random subset of the frames, while speckle noise was added to all frames. An example image (without specular noise) is shown in Figure 1. The Gibbs sampler is run with $N_{\text {burnin }}=100$ and $N_{\text {samples }}=20$. Performance is reported through the metrics:

- $E\left[\frac{\|D-\hat{D}\|_{1}}{\|D\|_{1}}\right]$ : The "foreground" error; i.e., the normalized expected error in the detection of pixels containing movers.

- $E\left[\frac{\|B-\hat{B}\|_{2}}{\|B\|_{2}}\right]$ : The "background" error; i.e., the normalized expected error in the reconstruction of the background (stationary) component.

Figures 2, 3, 4, 5 provide visualizations of the performance of the Gibbs sampler for varying number of passes, number of antenna, specular noise variance, and target variance, respectively. It is seen that as the number of observations increase (either through increasing the number of antenna or number of passes), the performance of 
the algorithm also increases, as expected. This is also shown to be true for the mover error as the detectability increases (by either increasing target variance or increasing the specular noise variance). Finally, the stationary error appears to be robust to the various values of specular and target variances.
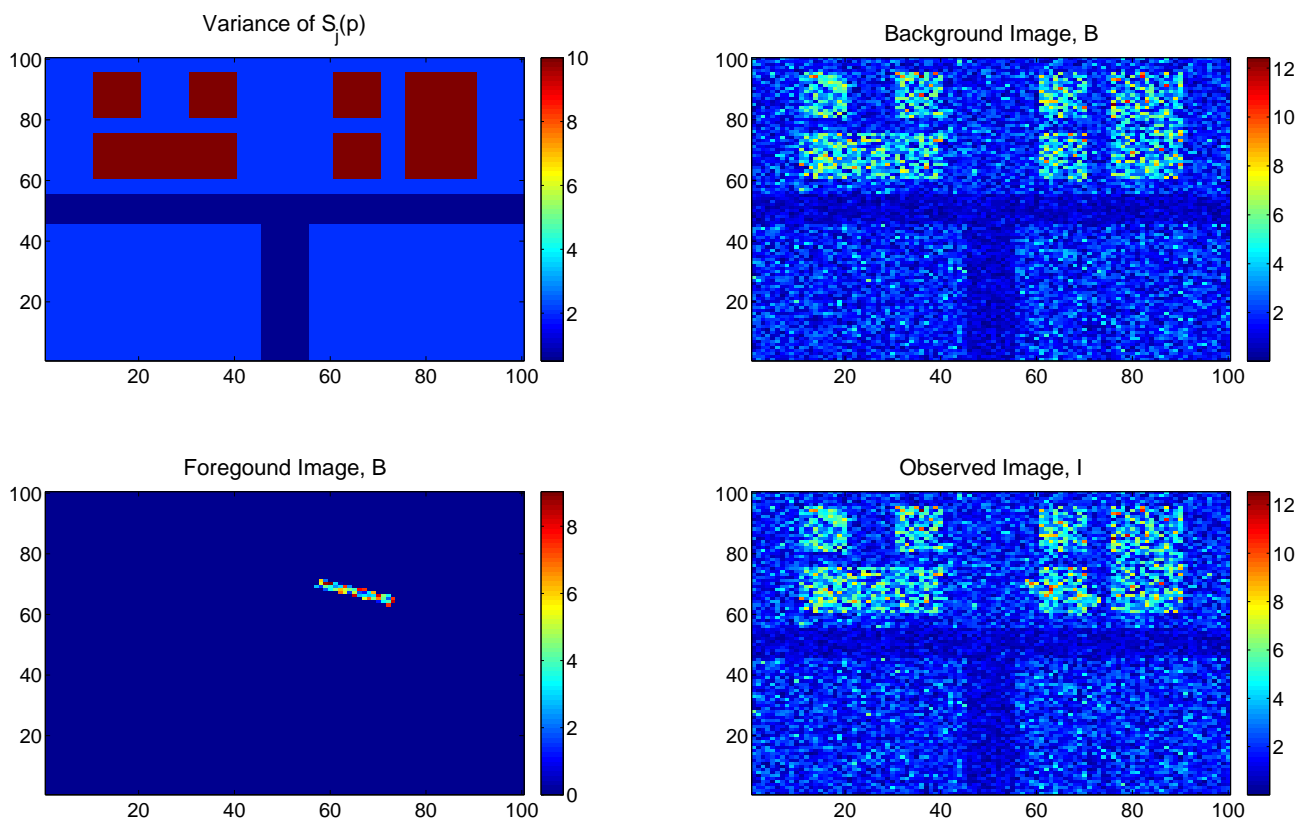

Figure 1. An example simulated image used for the performance analysis in section 6. In (a), the variance of the low-rank stationary component is shown for all pixels. In (b), a realization of the background image is shown. In (c), a realization of the foreground component is shown. Finally, in (d), a realization of the observed image (with background, foreground, additive noise, and reconstruction filter) is shown.

\subsection{Measured SAR imagery}

In this section, we present results of applying the hierarchical Bayesian algorithm to images created from the SAR GMTI Challenge Dataset. ${ }^{13}$ Images were created with 1 second integration length at 0.25 second increments per frame for a total of 8 seconds $(F=25)$. The dataset provides three antenna $(K=3)$ and two passes $(P=2)$. Once again, the Gibbs sampler was initialized with hyperparameter values suggested in the model description. Furthermore, the Monte Carlo inference parameters were set to $N_{\text {burnin }}=500$ and $N_{\text {samples }}=20$. Figure 6 provides the original data (1st column), the mean background component and foreground component from the Gibbs sampler (2nd and 3rd columns, respectively), and the foreground component from a standard coherent change detection (CCD) algorithm. It is seen that the Gibbs sampler output provides a significantly sparser output than the standard CCD algorithm. Moreover, the Gibbs sampler provides an explicit characterization of the background image, which is not provided by standard CCD.

\section{CONCLUSIONS AND FUTURE WORK}

In this paper we proposed a hierarchical Bayesian model for persistent SAR imagery, along with a Gibbs sampling scheme to efficiently estimate the posterior distribution. This algorithm can infer statistics of the noise without extensive tuning of hyperparameters, yet also provides a characterization of its uncertainty through a posterior distribution. Performance analysis with simulated imagery shows low reconstruction errors over a range of parameters, as well as robustness to noise parameters. Moreover, the proposed methods have been applied to images from measured SAR phase histories, yielding explicit characterizations of both the foreground (target 


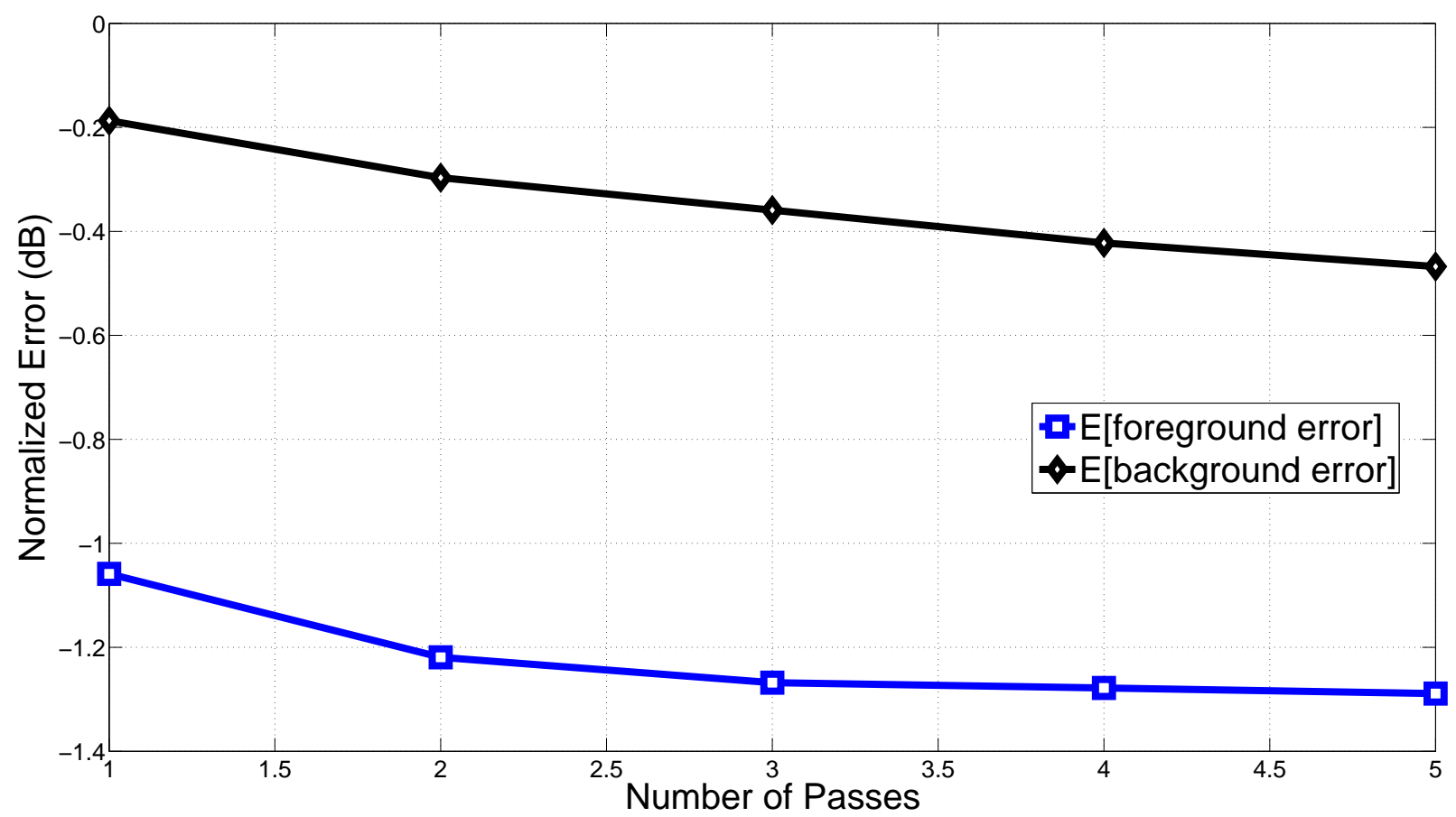

Figure 2. Normalized errors (dB scale) as a function of number of passes. It is seen that as the number of passes increase, the errors decrease.

signatures) and background components, as opposed to standard CCD which only provides the foreground component. Also, these methods are easily adaptable to further aspects of SAR imagery, including depthelevation maps, image registration errors, and target motion models.

Future work will include generalizing these methods to handle less restrictive assumptions on the observed images; in particular, the issue of errors in image registration must be accounted for explicitly. Furthermore, the Gibbs sampler is efficient for a fixed number of observations, but a sequential processing version for posterior estimation would be preferable in the case of streaming SAR imagery. Particle filters, for example, could be used as a replacement to the Gibbs sampler. Finally, foreground extraction is important for the ultimate goal of detecting and tracking targets. Accordingly, future work could fuse these methods with target tracking algorithms similar to previous work. ${ }^{4}$

\section{ACKNOWLEDGMENTS}

This research was partially supported by the AFRL ATR Center through a Signal Innovations Group subcontract, grant number SIG FA8650-07-D-1221-TO1.

\section{REFERENCES}

[1] X. Ding, L. He, and L. Carin, "Bayesian robust principal component analysis," submitted for publication, 2010.

[2] J. Jao, "Theory of synthetic aperture radar imaging of a moving target," IEEE Transactions on Geoscience and Remote Sensing, vol. 39, no. 9, pp. 1984-1992, 2001.

[3] J. Fienup, V. Syst, and M. Ann Arbor, "Detecting moving targets in SAR imagery by focusing," IEEE Transactions on Aerospace and Electronic Systems, vol. 37, no. 3, pp. 794-809, 2001.

[4] G. E. Newstadt, E. Zelnio, L. Gorham, and A. O. H. III, "Detection/tracking of moving targets with synthetic aperture radars," E. G. Zelnio and F. D. Garber, Eds., vol. 7699, no. 1. SPIE, 2010, p. 76990I. [Online]. Available: http://link.aip.org/link/?PSI/7699/76990I/1 


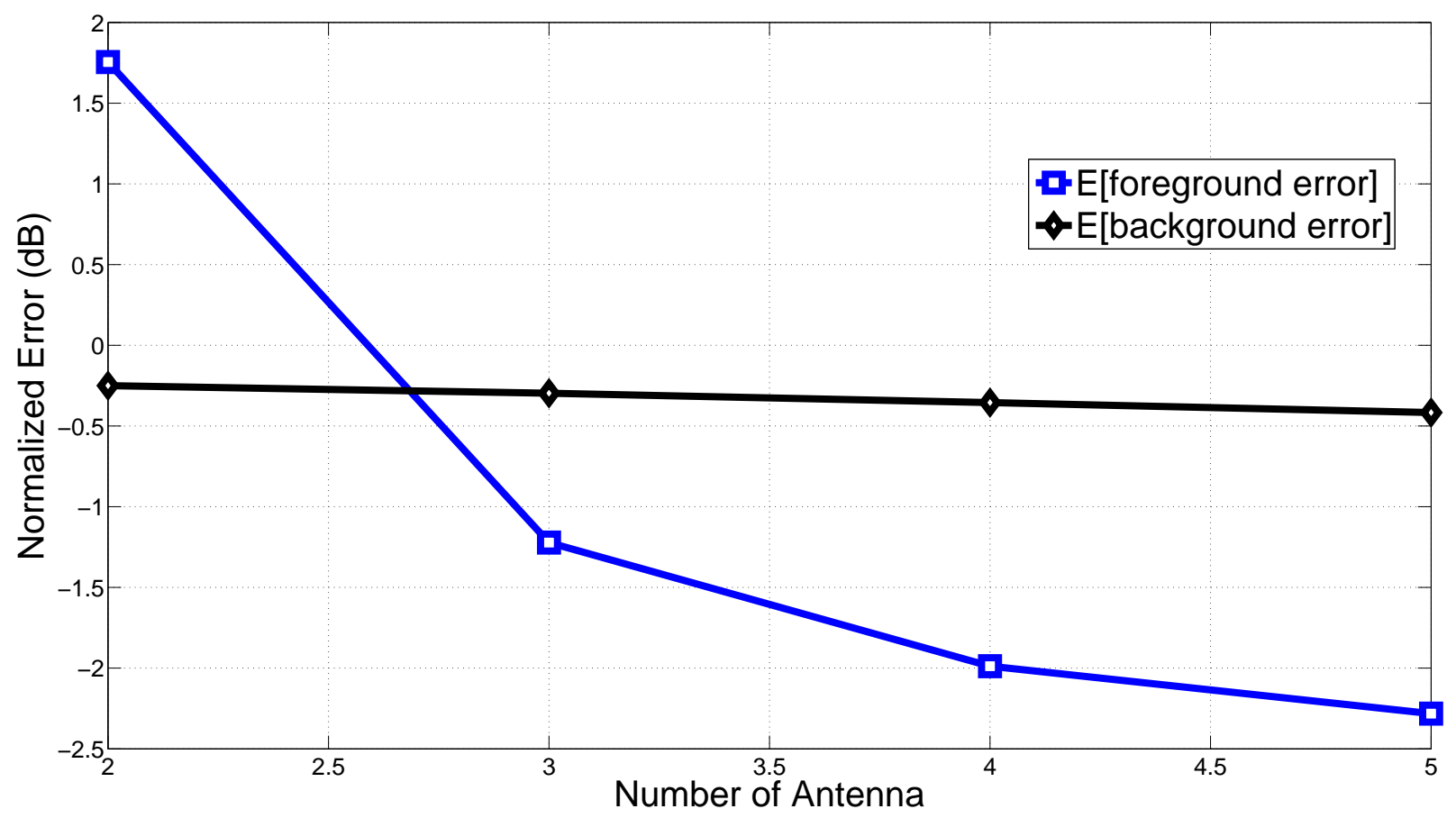

Figure 3. Normalized errors ( $\mathrm{dB}$ scale) as a function of number of antenna. It is seen that as the number of antenna increase, the errors decrease as expected. For 2 antenna, the normalized error is greater than 1, which suggests that too few antenna may be insufficient to reconstruct the subspace that the stationary component lies in.

[5] J. Ender, "Space-time processing for multichannel synthetic aperture radar," Electronics and Communication Engineering Journal, vol. 11, no. 1, pp. 29-38, 1999.

[6] M. Soumekh, "Moving target detection in foliage using along track monopulse synthetic aperture radar imaging," Image Processing, IEEE Transactions on, vol. 6, no. 8, pp. 1148 -1163, Aug. 1997.

[7] K. Ranney and M. Soumekh, "Signal subspace change detection in averaged multilook SAR imagery," Geoscience and Remote Sensing, IEEE Transactions on, vol. 44, no. 1, pp. 201 - 213, jan. 2006.

[8] B. Borden and M. Mumford, "A statistical glint/radar cross section target model," Aerospace and Electronic Systems, IEEE Transactions on, vol. AES-19, no. 5, pp. $781-785,1983$.

[9] E. Erten, A. Reigber, and O. Hellwich, "Aspects of multivariate statistical theory with the application to change detection," in Geoscience and Remote Sensing Symposium (IGARSS), 2010 IEEE International, 2010, pp. $1960-1963$.

[10] M. Tipping, "Sparse Bayesian learning and the relevance vector machine," The Journal of Machine Learning Research, vol. 1, pp. 211-244, 2001.

[11] S. Ji, Y. Xue, and L. Carin, "Bayesian compressive sensing," Signal Processing, IEEE Transactions on, vol. 56, no. 6, pp. 2346-2356, 2008.

[12] D. Wipf, J. Palmer, and B. Rao, "Perspectives on sparse Bayesian learning," in Advances in Neural Information Processing Systems 16: Proceedings of the 2003 Conference, vol. 16. The MIT Press, 2004, p. 249.

[13] S. M. Scarborough, C. H. Casteel Jr, L. Gorham, M. J. Minardi, U. K. Majumder, M. G. Judge, E. Zelnio, M. Bryant, H. Nichols, and D. Page, "A challenge problem for SAR-based GMTI in urban environments," E. G. Zelnio and F. D. Garber, Eds., vol. 7337, no. 1. SPIE, 2009, p. 73370G. [Online]. Available: http://link.aip.org/link/?PSI/7337/73370G/1 


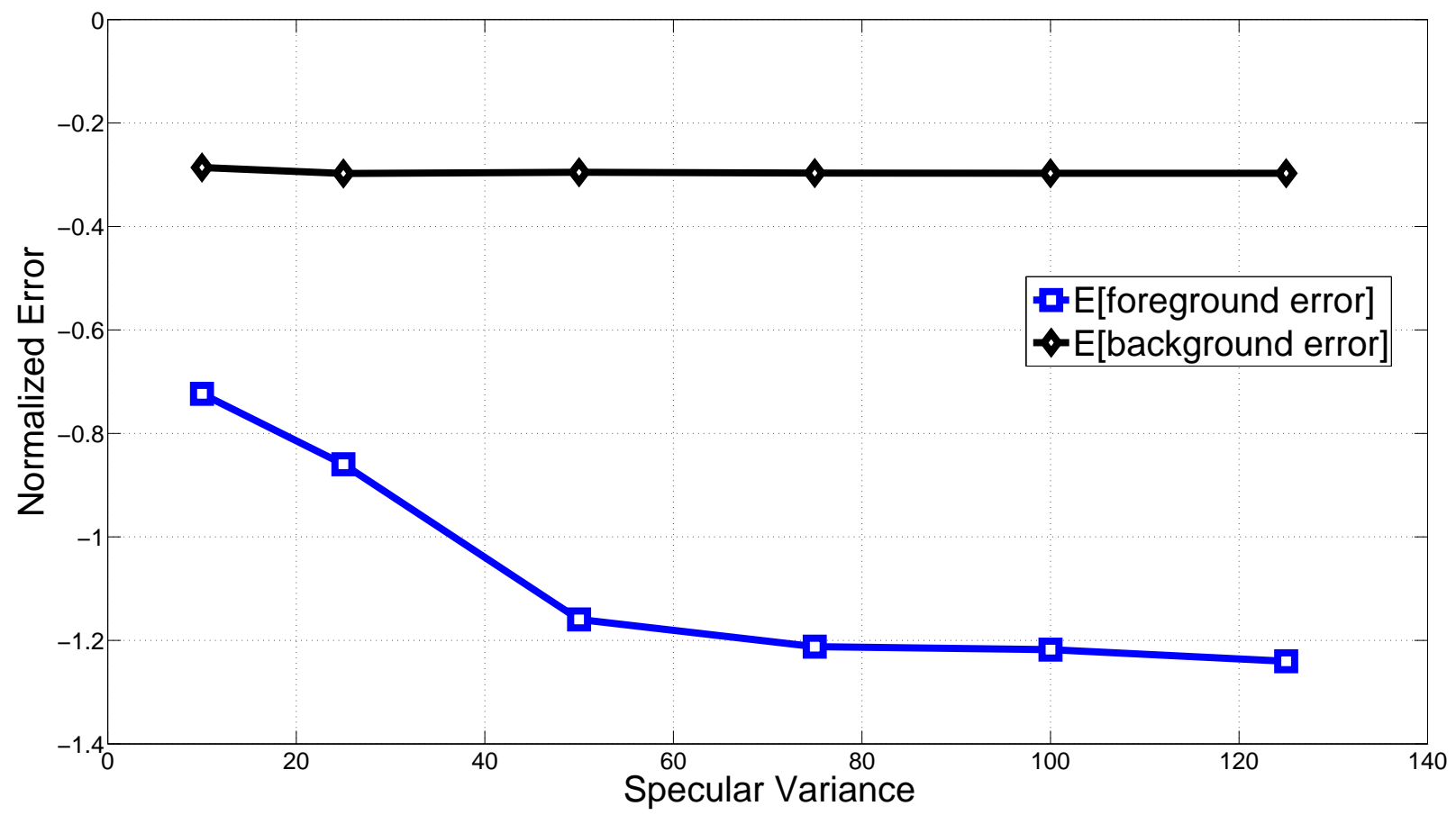

Figure 4. Normalized errors (dB scale) as a function of the specular variance. For low values of specular variance, it is more difficult to distinguish movers from specular noise, which causes larger errors in the reconstruction. However, for sufficiently large specular variance ( $>40$ in these simulations), the performance is invariant to specular variance, which suggests some measure of robustness to this error source. This is more apparent with the stationary error, which is nearly constant over all values of the specular variance. 


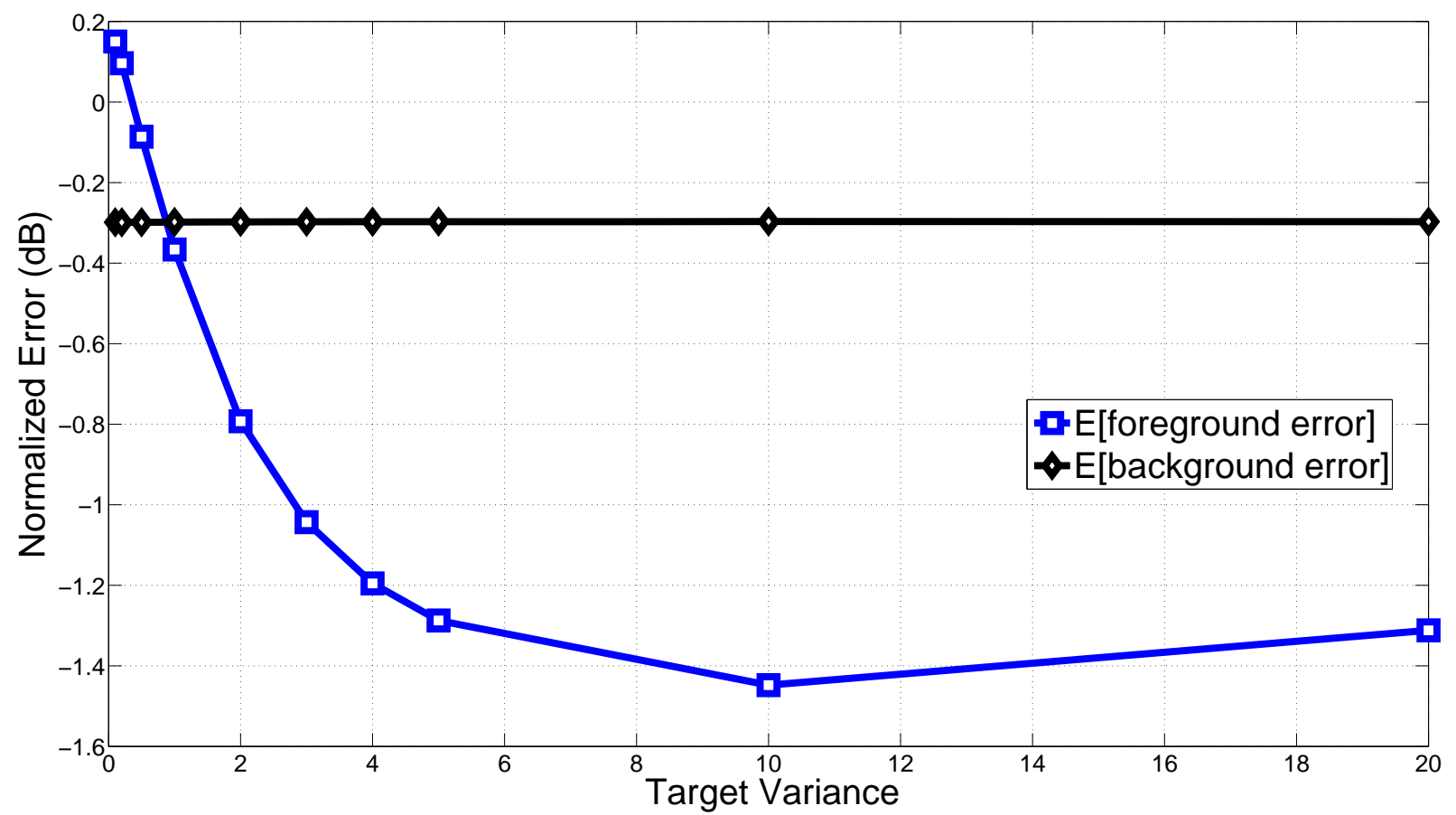

Figure 5. Normalized errors ( $\mathrm{dB}$ scale) as a function of the target variance. For low values of target variance, targets are indistinguishable from the noise sources, leading to poor detection performance. Conversely, target detection (in terms of mover error) improves as the target variance increases. Furthermore, the stationary error, which does not depend on the movers, is invariant to the target variance. 

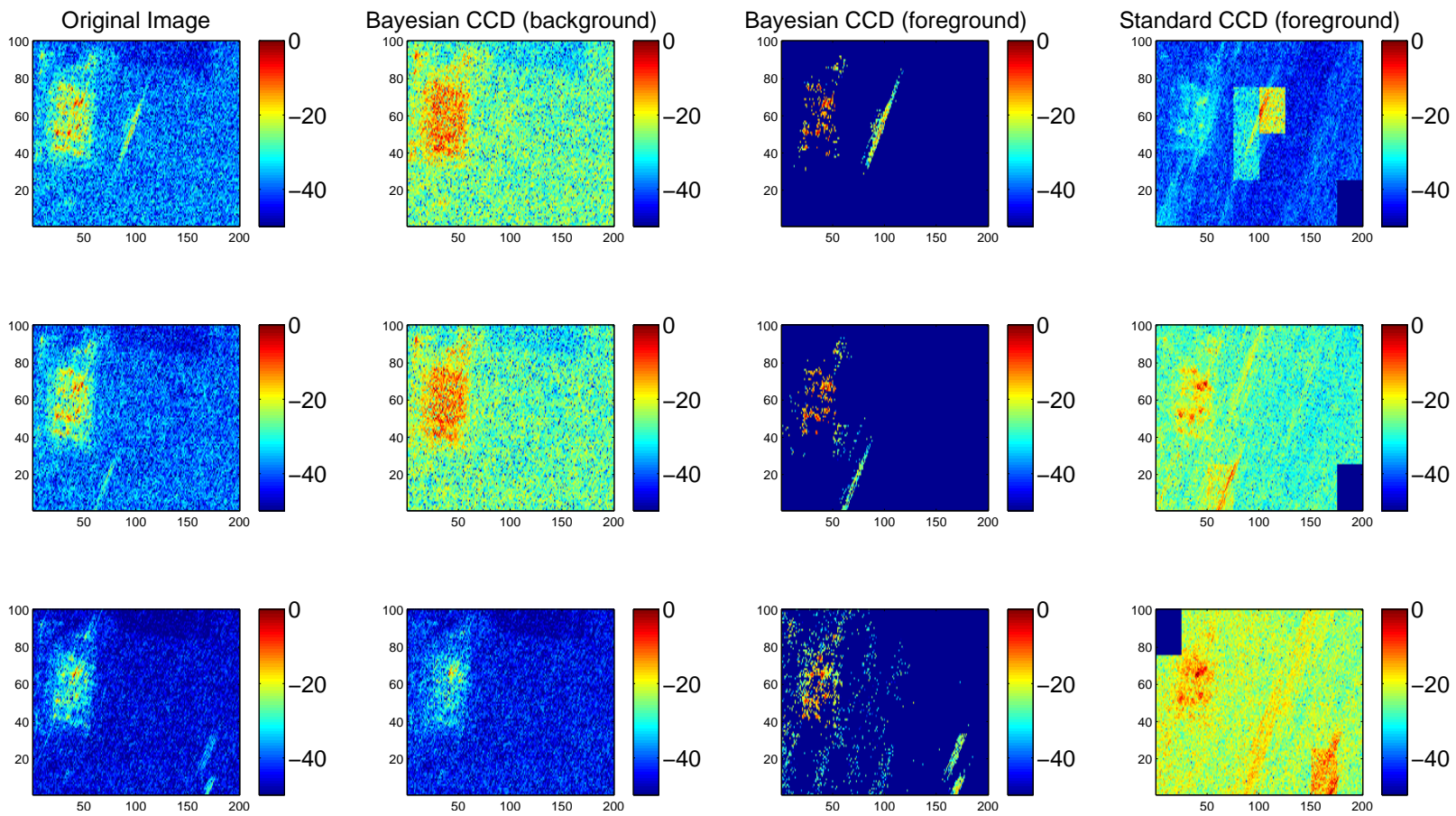

Figure 6. Measured SAR imagery from the SAR GMTI Challenge Dataset (1st column), the mean background component and foreground component from the output of the Gibbs sampler (2nd and 3rd columns, respectively), and the foreground component from a standard coherent change detection (CCD) algorithm. It is seen that the Gibbs sampler output provides a significantly sparser output than the standard CCD algorithm. Moreover, the Gibbs sampler provides an explicit characterization of the background image, which is not provided by standard CCD. 